\title{
Influences on allergic mechanisms through gut, lung, and skin microbiome exposures
}

\author{
Andrea M. Kemter and Cathryn R. Nagler \\ Department of Pathology and Committee on Immunology, The University of Chicago, Chicago, Illinois, USA.
}

\begin{abstract}
In industrialized societies the incidence of allergic diseases like atopic dermatitis, food allergies, and asthma has risen alarmingly over the last few decades. This increase has been attributed, in part, to lifestyle changes that alter the composition and function of the microbes that colonize the skin and mucosal surfaces. Strategies that reverse these changes to establish and maintain a healthy microbiome show promise for the prevention and treatment of allergic disease. In this Review, we will discuss evidence from preclinical and clinical studies that gives insights into how the microbiota of skin, intestinal tract, and airways influence immune responses in the context of allergic sensitization.
\end{abstract}

Allergic disease has been on the rise in Western societies in recent decades. Twin studies have shown that while genetic predisposition influences the development of allergic sensitization, environmental factors also play a very important role (1-4). Thirty years ago, the hygiene hypothesis postulated that microbial infections protect from allergy (5). This hypothesis has since been updated to encompass the commensal microbiota (6-8), based on the identification of bacteria that are allergy protective as well as the presence of potentially harmful bacteria that can drive allergic disease. The composition of the commensal microbiome is affected by multiple environmental factors, including the mode of delivery during childbirth (9), breast versus formula feeding $(10,11)$, a "Western diet" low in fiber and high in fat content $(12,13)$, and misuse of antibiotics (14).

Atopic diseases can take a number of different forms. Often, skin manifestations like atopic dermatitis ( $\mathrm{AD}$; or eczema) appear in infancy and can persist into adulthood. It is estimated that AD affects up to $20 \%$ of the population, especially in developed countries (15), and predisposes children to the development of food allergies in early childhood and to asthma by school age (16-20). This disease progression is often called the atopic or allergic march. Although the exact mechanisms leading to sensitization (instead of tolerance) to environmental antigens are still being investigated, a picture of the underlying immune response has emerged (for recent reviews, see refs. 6, 21, 22). Generally, production of the alarmins IL-25, IL-33, and thymic stromal lymphopoietin (TSLP) by epithelial cells activates local immune cells including basophils and type 2 innate lymphoid cells (ILC2s) (23). These cells then produce the type 2 cytokines IL-4, IL-5, and IL-13, which prime DCs to induce the differentiation of $\mathrm{CD}^{+} \mathrm{T}$ cells into Th2 cells (21-23). Cytokine-secreting Th2 cells activate allergen-specific B cells, inducing their class switch to $\operatorname{IgE}$ (22). The IgE isotype is a hallmark of allergic sensitization. Effector cells like mast cells, eosinophils, and basophils express IgE receptors. Aller-

Conflict of interest: CRN is president and cofounder of ClostraBio Inc.

Copyright: @ 2019 American Society for Clinical Investigation

Reference information: J Clin Invest. 2019;129(4):1483-1492.

https://doi.org/10.1172/JCI124610. gens cross-link IgE bound to these receptors and induce effector cell degranulation, causing the release of allergic mediators like histamine and eicosanoids like prostaglandins or leukotrienes, as well as proteases and cytokines. These mediators induce the typical symptoms of allergic reactions: vasodilation, smooth muscle hypercontractility, and mucus overproduction at mucosal sites, resulting in a broad range of clinical signs and symptoms, from redness and itching to deadly anaphylactic responses $(21,22)$.

In this Review, we will introduce the microbiota of skin, intestinal tract, and airways; describe their roles in health; and discuss findings on how alterations in their composition can affect mechanisms of allergic sensitization.

\section{The skin microbiota and atopic dermatitis}

Healthy human skin forms a barrier between our tissues and the environment. The top layer of the epidermis, the stratum corneum, consists of cross-linked keratinocytes that are constantly being shed. The skin surface is a hostile environment, desiccated with a high salt concentration and low $\mathrm{pH}$, although specific body sites differ in variables like moisture, lipid content, UV exposure, and temperature $(24,25)$. The composition of the skin microbiota depends on genetics, environmental factors, and the local microenvironment of the body site (26-29). In moist skin sites, corynebacteria and some staphylococci dominate, while in sebaceous (oily) areas lipophilic bacteria prevail, with propionibacteria and staphylococci being the most abundant; dry skin sites are colonized with mixed bacterial populations with abundant Betaproteobacteria and Flavobacteriales (27-29). Additional factors that determine the composition of the skin microbiome include the host immune system as well as competition for niche between the commensal bacteria themselves (Figure 1A). The skin is rich in antimicrobial lipids and antimicrobial peptides (AMPs), both of which are constitutively expressed or induced by the presence of specific microbes; commensal bacteria can produce AMPs as well (30-32). Commensal bacteria are also crucial for the education of the skin immune system, inducing the influx of microbiota-specific regulatory and effector T cells, which are important to control subsequent challenges with potential pathogens (33-35). Skin microbiota composi- 
A

B

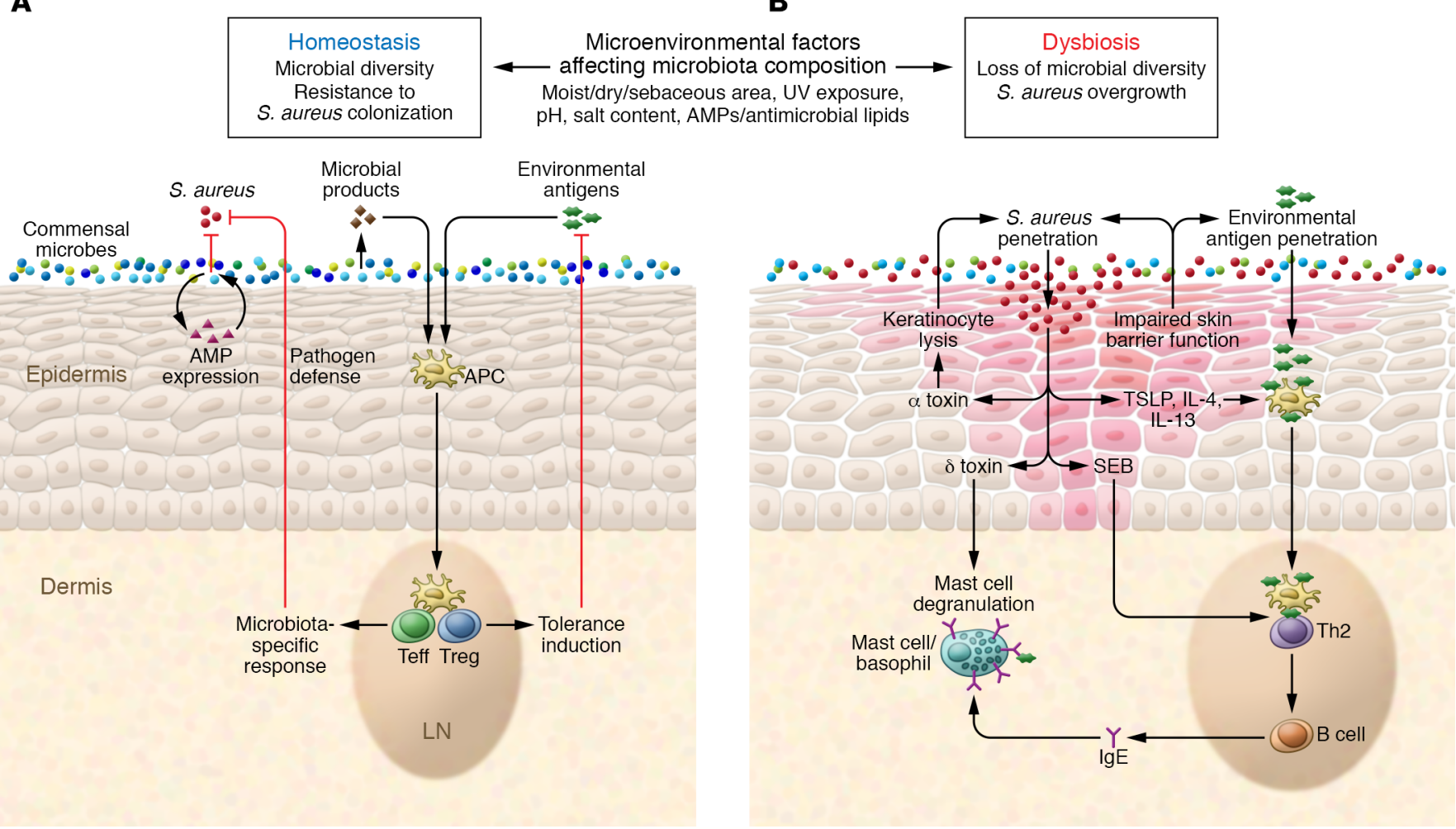

Figure 1. Skin commensals and an intact skin barrier promote tolerance induction, while skin barrier impairment and dysbiosis can drive type 2 inflammation. (A) In healthy individuals, the skin is colonized with a variety of skin commensals. Their composition depends on the local microenvironment and is balanced by antimicrobial peptides and lipids produced by the host or the commensals themselves. They also protect the host from colonization with potentially pathogenic bacteria. Colonization with beneficial commensals further induces local immune responses that protect the host from pathogens and promote induction of tolerance to environmental antigens. (B) Impaired skin barrier function is one of the main predisposing factors for atopic dermatitis (AD) development. Loss of microbial diversity as well as an overabundance of Staphylococcus aureus is also a common phenomenon on lesional skin of AD patients. Potentially pathogenic $S$. aureus strains can produce a number of molecules aiding in penetration of the host skin and driving type 2 immune responses, thereby exacerbating AD. Environmental antigens taken up through the inflamed skin are encountered in a pro-type 2 environment, which can lead to allergic sensitization with production of antigen-specific IgE. AMP, antimicrobial peptide; APC, antigen-presenting cell; LN, Iymph node; SEB, staphylococcal enterotoxin B; Teff, effector T cell; Treg, regulatory T cell; TSLP, thymic stromal lymphopoietin.

tion undergoes changes during a person's lifetime due to alterations in host biology. While the infant microbiome is fairly homogeneous across body sites at the time of delivery, it diversifies according to body site microenvironments within the first 6 weeks of life (36), and then changes during puberty as hormones affect the skin (37). The adult skin microbiome appears to be fairly stable over time (38). These findings demonstrate how environmental factors, host biology, and microbiota influence each other to establish a healthy skin microenvironment; the differences described between body sites or in different age groups may at least partially explain why certain skin conditions, including $\mathrm{AD}$, preferentially appear in certain age groups or at specific body sites.

Several different but interdependent factors seem to predispose patients to AD. First, in contrast to healthy skin, the barrier function of $\mathrm{AD}$ patients' skin is impaired (15). A number of genetic risk factors have been identified that directly affect barrier integrity, like loss-of-function mutations in the protein filaggrin, which is important for the development of the stratum corneum, or mutations that affect skin immune responses $(39,40)$. Mice with a filaggrin loss-offunction mutation develop $\mathrm{AD}$-like skin inflammation, underscoring the importance of this protein (41). Second, dysbiosis of the skin microbiota with decreased colonization of beneficial commensals $(42,43)$ and an increased presence of the potentially pathogenic commensal Staphylococcus aureus is a common phenomenon in both patients with AD and murine models of disease (44-46). The exact cause and timing of when particular predisposing factors come into play are not completely understood. While overgrowth of S. aureus sometimes precedes the development of AD (47), this is not always the case (43). However, in at least a subset of patients, alterations of the skin microenvironment, due to an impairment of the skin barrier function, affect colonization with commensals and their tightly regulated interplay with host cells. Alterations in the microenvironment could, for example, include moisture levels, $\mathrm{pH}$, nutrient availability, or shifts in the production of AMPs. Potentially pathogenic strains of $S$. aureus exploit these conditions, leading to their overgrowth; the lesional skin is colonized with $S$. aureus in up to $90 \%$ of patients with $\mathrm{AD}(48)$. The proportion of $S$. aureus relative to other commensals is increased during flares (44), and higher density is associated with more severe AD (49). Increased colonization with $S$. aureus is associated with a lower diversity of the skin microbiome. Diversity decreases further during a flare and increases if $\mathrm{AD}$ is treated (44); in a murine model, treatment with antibi- 
otics targeting $S$. aureus partially restored microbial diversity and almost completely prevented dermatitis development (45). Several studies have demonstrated how a diverse microbiome can keep the growth of $S$. aureus in check. Culturable Gram-negative bacteria from healthy human skin, for example, were able to enhance barrier function, control $S$. aureus colonization, and even alleviate dermatitis in a mouse model of $\mathrm{AD}$, unlike their counterparts from the skin of patients with AD (42). Certain strains of Staphylococcus (including S. epidermidis and S. hominis) produce AMPs that are active against S. aureus and even synergize with human AMPs. These strains are commonly found on healthy skin but are rare on patients with $\mathrm{AD}$ and, when reintroduced, acted against colonization with $S$. aureus $(31,32,50)$. S. epidermidis additionally produces a small lipopeptide that signals through host TLR2 and induces the production of AMPs by keratinocytes $(51,52)$. These findings demonstrate how loss of beneficial commensals and the AMPs they produce (or whose production they induce in host cells) enables $S$. aureus overgrowth, paving the way for establishment or exacerbation of AD.

What makes $S$. aureus so dangerous? The answer lies in the variety of factors $S$. aureus strains produce to exploit a weakened skin barrier and activate deleterious host immune reactions (Figure 1B). For instance, $S$. aureus produces a protease that enables it to penetrate into the dermis of $\mathrm{AD}$ patients or mice with filaggrin loss-of function mutations $(53,54)$. S. aureus penetration results in increased production of type 2 cytokines such as TSLP, IL-4, and IL-13 (53). S. aureus strains also produce a number of molecules that induce skin inflammation and exacerbation of AD. Among these are $\alpha$-toxin, which lyses keratinocytes, especially in the presence of type 2 cytokines $(55,56)$. Local concentrations of type 2 cytokines are increased by $\delta$-toxin, which induces mast cell degranulation (48), as well as by lipoproteins, $S$. aureus cell wall components that signal through TLR2/6 and induce TSLP production in keratinocytes (57). Staphylococcal enterotoxin B (SEB) is a superantigen that induces a mixed Th1/Th2 response after application to tape-stripped mouse skin and results in specific IgE responses to both SEB and the coapplied model antigen OVA (58). When applied to human skin, SEB led to skin inflammation in both healthy subjects and those with $\mathrm{AD}$; in three of six participants with $\mathrm{AD}$, the application of SEB led to a disease flare (59). All of these factors contribute to the exacerbation of $\mathrm{AD}$ by creating local inflammation and inducing a further breakdown of the skin barrier. In future studies, it will be interesting to further clarify the role of commensal bacteria in the development of $\mathrm{AD}$. What other factors might contribute to disease in those children who did not exhibit dysbiosis or an overgrowth of $S$. aureus before the development of AD? Current treatment regimens still mainly focus on suppressing inflammation and supporting the epidermal barrier with emollients (15). Combining these with therapies targeting the skin microbiome may be beneficial.

\section{$A D$ and allergic sensitization}

Skin inflammation and impaired barrier function likely contribute to allergic sensitization to environmental antigens. Several studies have shown that children with an impaired skin barrier or AD are at higher risk of atopic sensitization $(16-18,60,61)$. In fact, the outcomes of potential allergen application onto intact versus damaged skin have been compared to determine the best approach to epicutaneous immunotherapy. Whereas application of the allergen (in this case peanut extract) to intact skin induced tolerance, application to tape-stripped skin exacerbated the allergic response to peanut protein (62). In infants, application of peanut oil-containing creams to inflamed skin may lead to sensitization to peanut (63). In a mouse model, sensitization with peanut extract or OVA through $\mathrm{AD}$-like skin lesions increased basophil accumulation in the skin in a TSLP-dependent manner, inducing a systemic Th2 response with elevated levels of allergen-specific IgE (64). Similarly, mice sensitized through barrier-impaired skin exhibited airway allergic responses to OVA that depended on keratinocyte production of $\operatorname{TSLP}(65,66)$; in a separate study, coadministration of intradermal TSLP with OVA was sufficient to induce OVA-specific sensitization and airway allergic responses (67). In addition, mice with a filaggrin loss-of-function mutation developed spontaneous lung inflammation with increased airway hyperresponsiveness (41). These data suggest that antigen taken up through barrier-defective, inflamed skin can be inappropriately recognized by the immune system and elicit an antigen-specific Th2 response, resulting in allergic sensitization. This could at least partially explain the correlation between $\mathrm{AD}$ in early childhood and the subsequent development of food allergies or asthma. However, the microbiome influences immune maturation in more general terms (discussed below), which could potentially also influence predisposition to $\mathrm{AD}$ and certainly has effects on food allergies and asthma.

\section{The intestinal microbiota and food allergy}

Even more so than the skin, the intestinal tract is colonized with a large and diverse microbiota that is highly influenced by factors in the local microenvironment. The small and large intestines differ greatly in their morphology and function (see refs. 68 and 69 for review). In keeping with its functions in digestion and nutrient absorption, the surface area of the small intestine is enlarged by numerous villous projections; it is also covered with a fairly diffuse mucus layer. The mucus layer of the colon differs from that of the small intestine, having a two-layered structure with a dense and sterile inner layer covered by a more diffuse outer layer that is populated by bacteria (70). While the proximal small intestine is rich in easily accessible nutrients, nutrients are depleted along its length; similarly, concentrations of AMPs, oxygen, and bile acids (which are released into the proximal small intestine) as well as acidity decrease along the length of the intestinal tract $(68,69)$. Bacterial abundance increases from the duodenum to the terminal ileum and is highest in cecum and colon, which are enriched with anaerobic bacteria that ferment plant-derived dietary fibers $(69,71-73)$. Similarly, cross-sectional differences exist between regions closest to the epithelium, the mucus layer, and the lumen itself (74).

A number of studies have examined the intestinal bacterial composition of humans as well as mice. While there is large interindividual variability, Firmicutes and Bacteroidetes dominate overall $(71,74-77)$. However, like the skin microbiota, the intestinal microbiota changes over the course of an individual's life. The infant gut microbiome is shaped by factors like mode of birth (78, 79), maternal antibodies found in breast milk (80), and the type of oligosaccharides in the milk an infant is fed (81). The switch to solid foods changes the composition again (79), and it does not stabilize until about 3 years of age $(78,82)$. The adult microbiome, while affected by diet $(83)$, is fairly stable over time $(84,85)$. 


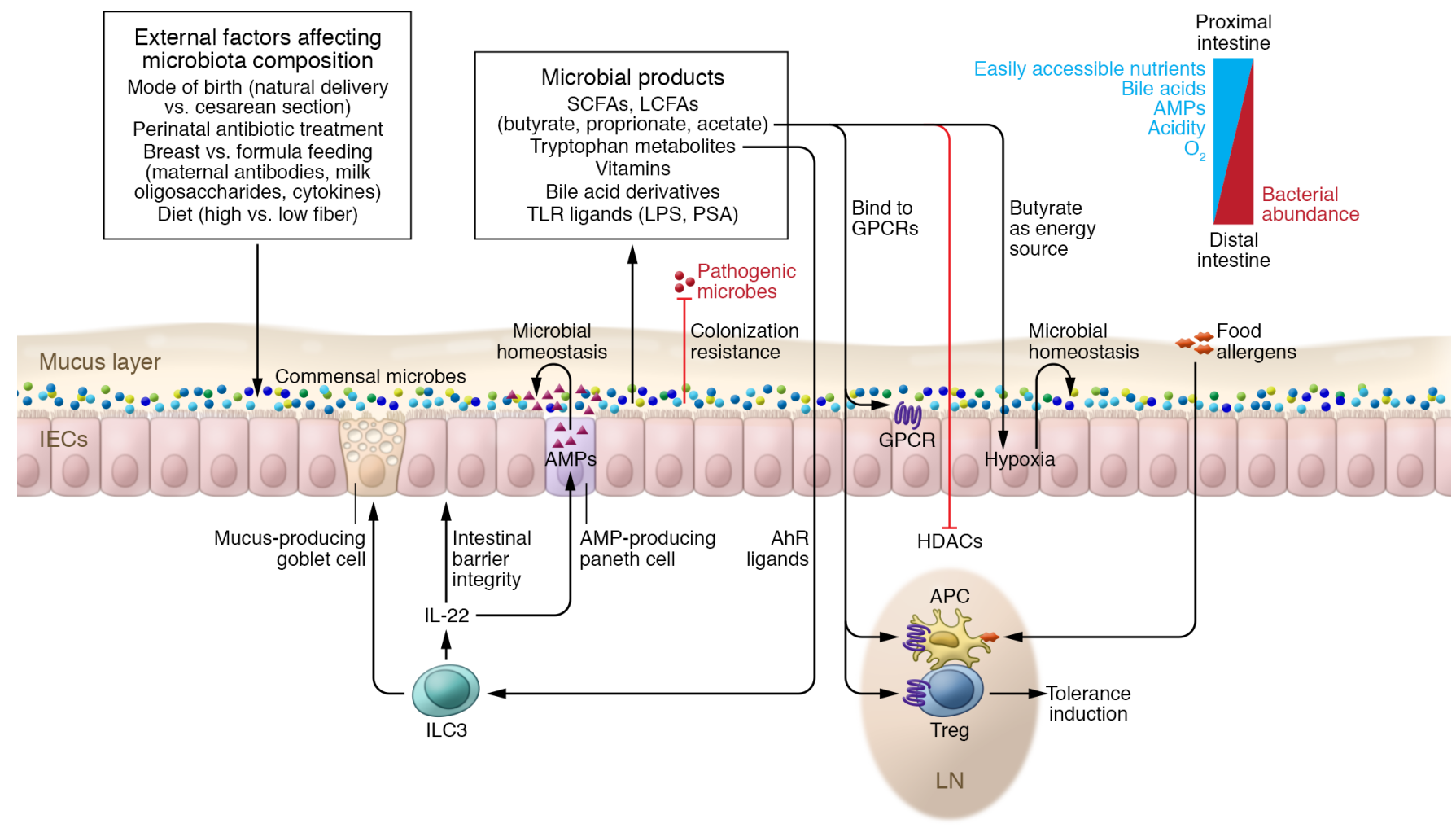

Figure 2. The healthy intestinal microbiota protects the intestinal barrier and promotes a tolerogenic microenvironment. A healthy gut microbiota containing fiber-fermenting anaerobic commensals can induce the differentiation of T cells into Tregs via several different mechanisms. SCFAs induce GPCR signaling as well as inhibit HDACs within DCs and T cells. Essential vitamins produced by bacteria can be important for Treg survival, and bile acids, which are modified by the intestinal microbiota, inhibit APC activation. In addition, molecules like PSA produced by Bacteroides fragilis can also induce Treg differentiation. SCFA production also protects the intestinal barrier. Butyrate, for example, is an important energy source for colonocytes; its utilization drives local hypoxia, which in turn favors the anaerobic microbiota. Tryptophan metabolites can act as AhR ligands and induce the production of IL-22 by ILC3s. IL-22 acts on the epithelium to strengthen the intestinal barrier function, for example by induction of antimicrobial peptides or mucus production. Dietary antigens sampled in this environment will lead to tolerance induction rather than sensitization against the antigen. AhR, aryl hydrocarbon receptor; AMP, antimicrobial peptide; APC, antigen-presenting cell; GPCR, G protein-coupled receptor; HDAC, histone deacetylase; IEC, intestinal epithelial cell; ILC, innate lymphoid cell; LCFA, long-chain fatty acid; LN, Iymph node; LPS, lipopolysaccharide; PSA, polysaccharide A; SCFA, short-chain fatty acid; Treg, regulatory T cell.

In addition to providing the host with protection from colonization with potential pathogens (see ref. 86 for a recent review on colonization resistance), symbiotic bacteria are important for intestinal barrier integrity as well as the development, education, and regulation of the immune system (Figure 2). Commensal bacteria induce the differentiation of colonic $\mathrm{T}$ cells into Tregs $(87,88)$. Specifically, Bacteroides fragilis polysaccharide A induces the differentiation of Tregs via TLR2 signaling $(89,90)$, while certain strains of Clostridia, mainly from clusters IV and XIVa, induce Tregs via TGF- $\beta(91,92)$. Commensals specifically expand a population of retinoic acid receptor-related orphan receptor (ROR) $\gamma t+$ colonic Tregs $(93,94)$ that keep Th2 responses in check (94). Short-chain fatty acids (SCFAs), produced by fermentation of dietary fiber by anaerobic bacteria, play an important role in the induction of Tregs (95). SCFAs can bind GPCRs (including GPR43, GPR41, and GPR109a) expressed on immune cells and the intestinal epithelium to induce Tregs and support epithelial homeostasis (95-98). The SCFAs butyrate and propionate also act as histone deacetylase (HDAC) inhibitors, leading to the acetylation of the Foxp3 coding region in T cells and increased expression of this transcription factor, driving Treg differentiation $(99,100)$ as well as reducing the production of proinflammatory mediators by DCs (99). Butyrate additionally serves as an energy source for colonocytes (101); it also induces beta-oxidation in colonocytes to keep the colonic environment hypoxic and enables the survival of beneficial obligate anaerobes (102). The picture emerging from these findings indicates a crucial role for dietary fiber-fermenting obligate anaerobes that, through production of SCFAs, strengthen intestinal barrier function and support the establishment of a generally tolerogenic environment by inducing Tregs as well as inhibiting proinflammatory DC functions. Other microbial metabolites also contribute to intestinal homeostasis. A long-chain fatty acid produced by lactobacilli signals through GPR40 and improves barrier function by inducing expression of tight junction proteins (103). Intestinal bacteria metabolize tryptophan to produce aryl hydrocarbon receptor (AhR) ligands that induce barrier-protective IL-22 expression (104). Bile acids are also metabolized by intestinal bacteria, altering their reabsorption and bioavailability. Bile acids inhibit antigen-presenting cell activation and reduce inflammation (105). In contrast to their hosts, certain commensal bacteria synthesize essential vitamins, some of which are important immunomodulators; one example of this is vitamin $\mathrm{B} 9$, which supports Treg survival (106-108). All of these factors likely contribute to the beneficial effects of a healthy intestinal microbiota. 
It is therefore not surprising that the intestinal microbiota contributes to the induction of tolerance against food antigens in mice $(109,110)$. When transferred into wild-type mice, the dysbiotic microbiota from allergy-prone IL4raF709 mice (with a gain of function mutation in the IL-4 receptor chain) increased susceptibility to oral allergic sensitization with OVA (111). Dysbiosis of the fecal microbiota in human infants is associated with food allergy (112-115). We found that, at just 4-5 months, the composition of the fecal microbiota of cow's milk-allergic (CMA) infants is strikingly different from age-matched healthy controls (113). We established a model for transfer of fecal bacteria from healthy and CMA infant donors into germ-free (GF) mice. We showed that GF mice develop an anaphylactic response to sensitization with the milk protein $\beta$-lactoglobulin (BLG). Colonization with feces from healthy infants protected the mice from an allergic response to BLG. In contrast, mice colonized with feces from infants with CMA exhibited an anaphylactic response to BLG sensitization and challenge. We were able to narrow this effect down to a single species of butyrateproducing Clostridia, Anaerostipes caccae, present in the healthy infant microbiota. Colonization of GF mice with A. caccae was sufficient to mimic the effects of the healthy microbiota and protect against an allergic response to BLG (116). Earlier work from our laboratory showed that colonization of GF or antibiotic-treated mice with a mixture of mouse-derived Clostridia strains also alleviated allergic sensitization in a peanut allergy model (117). In agreement with previous studies $(91,92)$, Clostridia colonization increased colonic Treg populations (117). Mackay and colleagues showed that mice fed high fiber diets exhibited an increase in fiber-fermenting anaerobic bacteria producing SCFAs with increased Tregs and enhanced tolerogenic capacity of DCs, all of which contributed to protection against allergic sensitization with peanut extract (118).

Colonization of GF or antibiotic-treated mice with Clostridia also improved intestinal barrier function in an IL-22-dependent manner (117), shedding light on another crucial mechanism supporting intestinal health. The clinical relevance of these findings is highlighted by the observation that intestinal barrier integrity is impaired in patients with food allergies (119). Impaired intestinal barrier integrity affects the access of allergen from the gut lumen to underlying immune cells. With an intact barrier, antigen is sampled by antigen-presenting cells and presented in a way that supports the induction of tolerance, while in individuals with impaired barrier function, antigens enter the systemic circulation as intact proteins capable of inducing an IgE response. We detected intact peanut allergens in the serum of GF or antibiotic-treated mice shortly after gavage of peanut extract, but not in Clostridia-colonized mice or mice that received an IL-22-Fc fusion protein (117).

Direct stimulation of immune cells by microbial components also influences susceptibility to allergic sensitization. For example, mice with mutations in TLR4, the receptor for LPS, showed increased concentrations of serum IgE as well as an exacerbated response to allergic sensitization with peanut extract (120), pointing to a protective role for TLR4 signaling. Indeed, a recent study comparing the intestinal microbiota of young children from Finland (who display a high prevalence of allergic sensitization) and Russia (who display a low prevalence) found a difference in the type of LPS expressed by bacteria abundant in these populations. Children from Finland had a high abundance of Bacteroides in their intestinal microbiota during the first 3 years of their lives, which affected innate immune signaling differently from the type of LPS produced by the intestinal bacteria of the Russian children (121). In future studies it will be interesting to explore how stimulation of immune cells, for example through TLR4, affects the intestinal immune environment in the context of food allergies. Does stimulation with immunogenic LPS induce a Th1 response and thereby a shift away from the type 2 response necessary for allergic sensitization? Or do certain bacterial populations present early in life prime the immune system to not react inappropriately later on, whenever it is confronted with microbial antigens or allergens?

\section{The intestinal microbiota and asthma}

Intestinal dysbiosis in early infancy is also associated with increased risk for later asthma development $(122,123)$. One study assessed asthma risk using positive skin prick testing and the presence of clinical wheeze at 1 year of age; the combination of those two factors was associated with a positive Asthma Predictive Index at age 3 and thereby an increased risk of having active asthma at school age (123). The authors described a decrease in the genera Rothia, Faecalibacterium, Lachnospira, and Veillonella (the latter three of which are Firmicutes and known SCFA producers) as well as a reduction in fecal acetate in those infants with an elevated risk of becoming asthmatic (123). A reduction in Faecalibacterium was also associated with an increased risk of atopic sensitization and asthma development in a separate study (122). Colonization of GF mice with Rothia, Faecalibacterium, Lachnospira, and Veillonella alleviated allergic airway inflammation (AAI) in OVA/alum-sensitized adult offspring of the colonized mice. These mice exhibited reduced histopathology scores and reduced numbers of immune cells detectable in the bronchoalveolar lavage fluid after intranasal challenge with OVA (123). Earlier work demonstrated exacerbated AAI in a similar OVA/alum airway hypersensitivity model in mice deficient in the acetate-binding GPR43 when compared with WT mice (97). However, signaling through GPR43 is not the only mechanism of action described for acetate. In mice that were intranasally sensitized and challenged with house dust mite extract, high-fiber diet led to an increase in intestinal acetate that induced Treg differentiation and ameliorated AAI via inhibition of HDACs (124). Furthermore, high-fiber diets induced intestinal bacterial production of propionate, which acted on DC and macrophage precursors in the mouse bone marrow and led to lung DCs being more phagocytic and less able to induce Th2 responses, again ameliorating AAI (96). Finally, butyrate was recently shown to inhibit ILC2 proliferation as well as IL-5 and IL-13 production, likely through its ability to act as an HDAC inhibitor, and thereby ameliorate Alternaria alternata-induced AAI (125).

These studies further emphasize the influence of the microbiome on systemic aspects of the immune response. Alterations in intestinal microbiota composition and metabolite production affected not only bone marrow precursors of immune cells (96), but also gene expression in the lung itself (124). These changes in turn favored the establishment of type 2 responses against aeroallergens over tolerance induction in airways (Figure 3).

\section{The airway microbiota and allergic asthma}

Unlike the skin and intestinal tract, the airways have typically been thought to be sterile, since culture-dependent methods failed to 


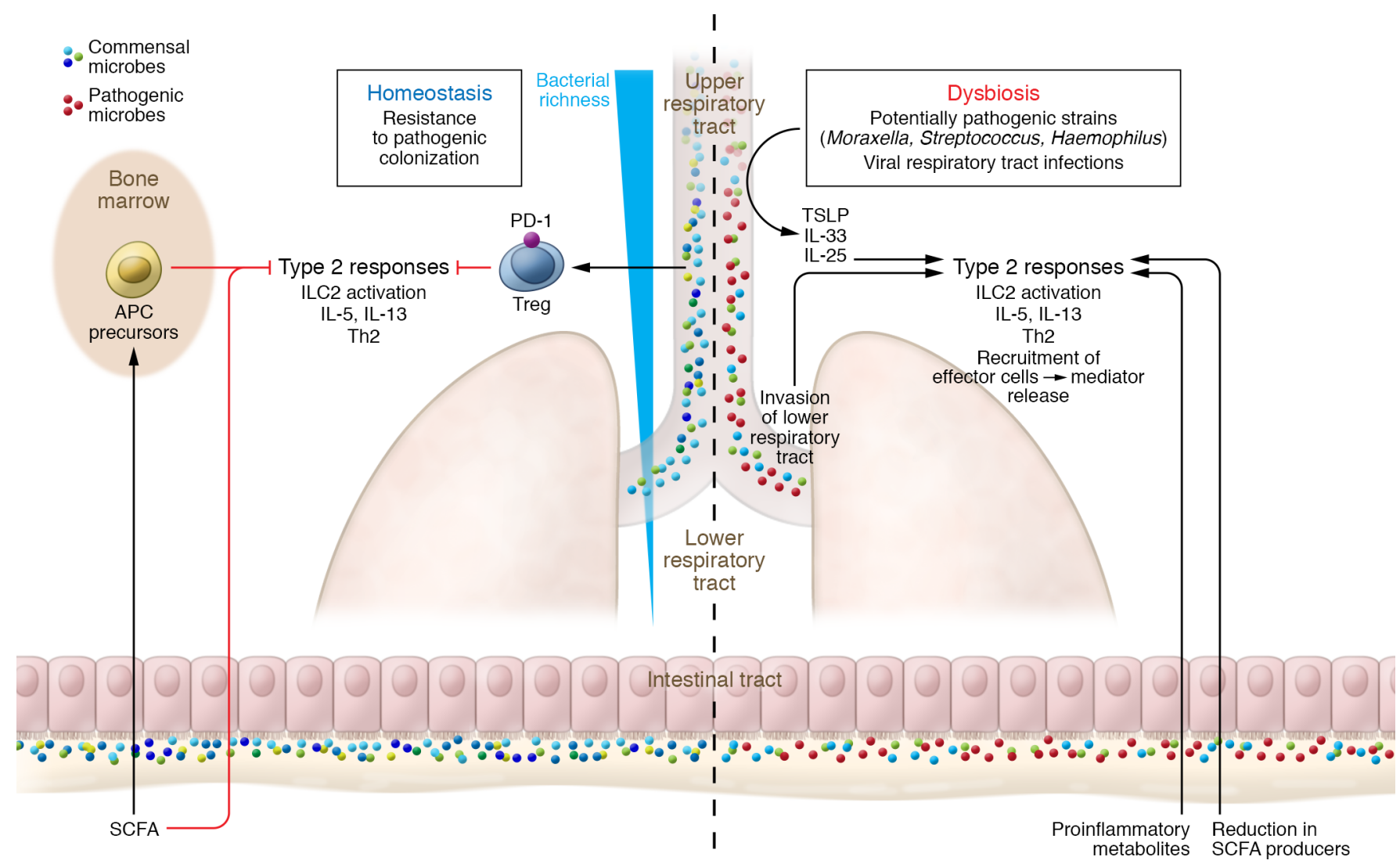

Figure 3. Exposure to a healthy microbiota drives antiinflammatory homeostatic conditions in the airways, while dysbiosis and viral respiratory tract infections can induce type $\mathbf{2}$ inflammation. It has become apparent in recent years that bacteria can also be found in a healthy respiratory tract, with decreasing community richness in the lower airways. Commensal colonization induces the differentiation of peripheral Tregs that are crucial for the control of type 2 immune responses. The presence of Proteobacteria increases the risk of developing asthma, partly as a result of an increased risk for viral infections, particularly in the lower airways. Viral infections induce the release of the alarmins TSLP, IL-33, and IL-25 from the airway epithelium, inducing type 2 inflammation. In addition, intestinal microbiome homeostasis influences airway immune responses. Metabolites like SCFAs can act systemically, for example affecting APC precursors in the bone marrow to give rise to APCs populating the airways that are less capable of inducing type 2 responses. On the other hand, proinflammatory metabolites of a dysbiotic intestinal microbiota can play a role in driving inappropriate immune responses. APC, antigen-presenting cell; ILC, innate lymphoid cell; SCFA, short-chain fatty acid; Treg, regulatory T cell; TSLP, thymic stromal lymphopoietin.

identify any resident microbiota. The introduction of cultureindependent methods of analysis like $16 \mathrm{~S}$ rRNA targeted sequencing has changed this view, although the existence of a resident lung microbiota remains controversial $(126,127)$. Owing to very high interindividual variability, there is little agreement on what constitutes a healthy airway microbiome; members of the Proteobacteria, Firmicutes, Actinobacteria, and Bacteroidetes phyla have all been identified $(128,129)$. In general, the microbiome composition of the upper airway seems very similar to that of the lung, although the community richness decreases in the lower airways $(130,131)$. The composition also appears to be fairly stable over time aside from increasing in richness over the first 2 years of a child's life $(132,133)$.

Numerous studies have explored the association of microbial dysbiosis in the airways and sensitization to aeroallergens and development or exacerbation of asthma (127, 134-137). In general, it appears that asthmatics' airway microbiomes are enriched in Proteobacteria, while the airways of healthy individuals show a higher abundance of Bacteroidetes and Firmicutes (127, 134-137). Three genera of Proteobacteria (Moraxella, Streptococcus, and Haemophilus) were consistently associated with increased risk of develop- ing asthma $(132,136,138-140)$. Colonization with these genera is associated with viral respiratory tract infections, which in turn have been associated with exacerbation of asthma (141); in addition, rhinovirus-induced wheezing in the first 3 years of life increases the risk of having asthma at age 6 (142) or 13 (143). This finding as well as rhinovirus-induced exacerbation of existing asthma can be explained, in part, by the virus's induction of IL-25, IL-33, and TSLP in airway epithelial cells, which led to expansion of ILC2s and increased airway hyperresponsiveness in mice (144-147). Furthermore, colonization with these potentially pathogenic Proteobacteria appears to promote the spread of viral respiratory tract infections into the lower airways, with the risk of developing asthma later on increasing the earlier an infant was first colonized $(132,138)$. These data suggest that early-life colonization with pathogens can impair appropriate maturation of the infant immune system and tolerance by inducing type 2 responses in the airways (Figure 3).

\section{The early-life window of opportunity}

The timing of microbial colonization or dysbiosis as well as exposure to potential allergens is important to consider (148). The evi- 
dence suggests that there is an early "window of opportunity" that is crucial for the maturation of the immune system. The effect of disruption of normal colonization during this critical time frame can be observed in humans, since dysbiosis in infants, for example due to several courses of antibiotics, is associated with the development of food allergies $(112,115,149)$. Increased production of IgE and exacerbated orally induced anaphylaxis or AAI in GF mice are normalized by colonization during early life, but not if colonization is delayed until adulthood (150-152). In addition, it has recently become clear that introduction of potentially allergenic foods early in infancy is actually beneficial rather than increasing the risk of becoming sensitized as initially thought (153-155).

These findings are of interest in the context of immune system development. During fetal development, both maternal and fetal immune reactions are carefully controlled to avoid Th1-mediated rejection of the fetus, both by production of type 2 cytokines and, more importantly, via regulatory immune responses $(8,156)$. In addition, the maternal microbiome has an important influence on fetal immune system development. Maternal IgG can cross the placenta, trafficking microbial components and metabolites to the fetus and thereby influencing fetal immune development (157). SCFAs can cross the placenta and promote an immunoregulatory environment via GPCR signaling and epigenetic effects (124). The proportion of Tregs is higher in mucosal sites during early infancy compared with later in life (158), and the immune system of newborns is Th2-skewed $(159,160)$, indicating that these in utero effects continue for a short while after birth. These mechanisms likely help prepare the developing fetus for its colonization with a diverse microbiota upon birth, which is further controlled by factors found in breast milk. The composition of the intestinal microbiota is influenced by maternal antibodies (80), by milk oligosaccharides that provide the main energy source for gut commensals (81), and even by bacteria found in breast milk (161). Other components of breast milk can affect infant immune development directly. These include allergy-protective polyunsaturated fatty acids as well as a variety of cytokines (162). TGF- $\beta$ is detectable in breast milk at concentrations reported to influence infant immune development $(162,163)$.

Considering all these factors, it therefore appears that appropriate immune responses to the colonizing microbiota are established under a tightly controlled regulatory environment, in which the Th2 skewing observed in newborns is balanced with an increase in type 1 responses. It is not surprising, then, that disruption of normal microbial colonization during this crucial developmental window enhances susceptibility to allergy. Several studies show that children who develop allergies later in life have an increase in proinflammatory responses as newborns $(164,165)$. Similar effects may be caused by proinflammatory metabolites of dysbiotic microbiota, which have been associated with an increased atopy risk (122).

It therefore appears that during a critical window early in life, the commensal microbiota creates microenvironments conducive to appropriate immune maturation and induction of tolerance toward ingested or environmental antigens; dysbiosis during this critical time period can disrupt this process, eventually resulting in the induction of allergic responses instead.

\section{Concluding remarks}

The findings presented in this Review emphasize the importance of a healthy microbiome for the prevention of allergic diseases. Some work suggests that the effects of the "Western lifestyle" can be partially alleviated by specific interventions. For example, vaginal microbiota transfer was recently shown to be at least partially successful in combating the influence of birth by cesarean section on the initial colonization of infants, although the long-term effects still remain to be seen (166).

The use of probiotics (living bacteria), prebiotics (foods to support the growth of beneficial bacteria), and synbiotics (both combined) is an intriguing possibility; however, research into the efficacy of these formulations is inconclusive $(156,167)$. The available data suggest that the efficacy of traditional probiotics is limited to the prevention of eczema in high-risk infants $(156,167,168)$. Fewer studies have investigated the efficacy of pre- or synbiotics, making it difficult to form conclusions $(156,167)$. Synbiotics may be a promising option. The importance of an adequate supply of nutrients for microbial growth and the production of beneficial metabolites can be seen, for example, in comparing effects of low- and high-fiber diets $(96,118,124)$. However, remaining questions on which bacterial strains to use, which prebiotics to add, and what dosage and what timing of administration to choose will have to be answered first. Furthermore, traditional probiotics are not FDA-approved drugs that can be sold as treatments for specific conditions; they are usually delivered as foods or as dietary supplements (169). In contrast, the development of live biotherapeutics in adherence with FDA regulations has garnered attention in recent years (169). Prime targets for these efforts include a number of dietary fiber-fermenting obligate anaerobes $(116,117$, $122,123)$. Another intriguing option currently being investigated is the direct use of bacterial products or their formulations; SCFAs are a focus for such research, considering their allergy-protective effects $(96,118,124,125)$. The combination of live biotherapeutics with active bacterial metabolites may be the best strategy for restoring an allergy-protective microbiota. Development of these therapeutics is still in the early stages but shows considerable promise for the treatment and prevention of allergic diseases.

\section{Acknowledgments}

We thank Evelyn Campbell and Elliot Culleen for critical review of the manuscript. This work was funded by the Sunshine Charitable Foundation and NIH grant AI106302.

Address correspondence to: Cathryn R. Nagler, The University of Chicago, 924 East 57th Street, JFK R410, Chicago, Illinois 60637, USA. Phone: 773.702.6317; Email: cnagler@bsd.uchicago.edu.
1. Strachan DP, Wong HJ, Spector TD. Concordance and interrelationship of atopic diseases and markers of allergic sensitization among adult female twins. J Allergy Clin Immunol. 2001;108(6):901-907.
2. Sicherer SH, Furlong TJ, Maes HH, Desnick RJ, Sampson HA, Gelb BD. Genetics of peanut allergy: a twin study. J Allergy Clin Immunol. 2000;106(1 pt 1):53-56.

3. Larsen FS, Holm NV, Henningsen K. Atopic der- matitis. A genetic-epidemiologic study in a population-based twin sample. JAm Acad Dermatol. 1986;15(3):487-494.

4. Liu X, et al. Genetic and environmental contributions to allergen sensitization in a Chinese twin 
study. Clin Exp Allergy. 2009;39(7):991-998.

5. Strachan DP. Hay fever, hygiene, and household size. BMJ. 1989;299(6710):1259-1260.

6. Reynolds LA, Finlay BB. Early life factors that affect allergy development. Nat Rev Immunol. 2017;17(8):518-528.

7. Rook GA, Lowry CA, Raison CL. Microbial 'old friends', immunoregulation and stress resilience. Evol Med Public Health. 2013;2013(1):46-64.

8. Jenmalm MC. The mother-offspring dyad: microbial transmission, immune interactions and allergy development. JIntern Med. 2017;282(6):484-495.

9. Dominguez-Bello MG, et al. Delivery mode shapes the acquisition and structure of the initial microbiota across multiple body habitats in newborns. Proc Natl Acad Sci U S A. 2010;107(26):11971-11975.

10. Praveen P, Jordan F, Priami C, Morine MJ. The role of breast-feeding in infant immune system: a systems perspective on the intestinal microbiome. Microbiome. 2015;3:41.

11. Fan W, et al. Diversity of the intestinal microbiota in different patterns of feeding infants by Illumina high-throughput sequencing. World J Microbiol Biotechnol. 2013;29(12):2365-2372.

12. De Filippo C, et al. Impact of diet in shaping gut microbiota revealed by a comparative study in children from Europe and rural Africa. Proc Natl Acad Sci U S A. 2010;107(33):14691-14696.

13. Sonnenburg ED, Smits SA, Tikhonov M, Higginbottom SK, Wingreen NS, Sonnenburg JL. Diet-induced extinctions in the gut microbiota compound over generations. Nature. 2016;529(7585):212-215.

14. Dethlefsen L, Huse S, Sogin ML, Relman DA. The pervasive effects of an antibiotic on the human gut microbiota, as revealed by deep $16 \mathrm{~S}$ rRNA sequencing. PLoS Biol. 2008;6(11):e280.

15. Weidinger S, Novak N. Atopic dermatitis. Lancet. 2016;387(10023):1109-1122.

16. Hill DJ, Sporik R, Thorburn J, Hosking CS. The association of atopic dermatitis in infancy with immunoglobulin E food sensitization. JPediatr. 2000;137(4):475-479.

17. Papathoma E, Triga M, Fouzas S, Dimitriou G. Cesarean section delivery and development of food allergy and atopic dermatitis in early childhood. Pediatr Allergy Immunol. 2016;27(4):419-424.

18. Brough HA, et al. Atopic dermatitis increases the effect of exposure to peanut antigen in dust on peanut sensitization and likely peanut allergy. JAllergy Clin Immunol. 2015;135(1):164-170.

19. van der Hulst AE, Klip H, Brand PL. Risk of developing asthma in young children with atopic eczema: a systematic review. JAllergy Clin Immunol. 2007;120(3):565-569.

20. Carlsten C, et al. Atopic dermatitis in a highrisk cohort: natural history, associated allergic outcomes, and risk factors. Ann Allergy Asthma Immunol. 2013;110(1):24-28.

21. Noti M. New perspectives on the initiation of allergic immune responses at barrier sites. Curr Opin Immunol. 2018;54:130-136.

22. Lambrecht BN, Hammad H. The immunology of the allergy epidemic and the hygiene hypothesis. Nat Immunol. 2017;18(10):1076-1083.

23. Hammad H, Lambrecht BN. Barrier epithelial cells and the control of type 2 immunity. Immunity. 2015;43(1):29-40.

24. Byrd AL, Belkaid Y, Segre JA. The human skin microbiome. Nat Rev Microbiol. 2018;16(3):143-155.

25. Chen YE, Fischbach MA, Belkaid Y. Skin microbiota-host interactions. Nature. 2018;553(7689):427-436.

26. Oh J, et al. Biogeography and individuality shape function in the human skin metagenome. Nature. 2014;514(7520):59-64.

27. Costello EK, Lauber CL, Hamady M, Fierer N, Gordon JI, Knight R. Bacterial community variation in human body habitats across space and time. Science. 2009;326(5960):1694-1697.

28. Findley K, et al. Topographic diversity of fungal and bacterial communities in human skin. Nature. 2013;498(7454):367-370.

29. Grice EA, et al. Topographical and temporal diversity of the human skin microbiome. Science. 2009;324(5931):1190-1192.

30. Nagy I, et al. Propionibacterium acnes and lipopolysaccharide induce the expression of antimicrobial peptides and proinflammatory cytokines/ chemokines in human sebocytes. Microbes Infect. 2006;8(8):2195-2205.

31. Cogen AL, et al. Selective antimicrobial action is provided by phenol-soluble modulins derived from Staphylococcus epidermidis, a normal resident of the skin. J Invest Dermatol. 2010;130(1):192-200.

32. Nakatsuji $T$, et al. Antimicrobials from human skin commensal bacteria protect against Staphylococcus aureus and are deficient in atopic dermatitis. Sci Transl Med.2017;9(378):eaah4680.

33. Naik S, et al. Commensal-dendritic-cell interaction specifies a unique protective skin immune signature. Nature. 2015;520(7545):104-108.

34. Naik S, et al. Compartmentalized control of skin immunity by resident commensals. Science. 2012;337(6098):1115-1119.

35. Scharschmidt TC, et al. A wave of regulatory T cells into neonatal skin mediates tolerance to commensal microbes. Immunity. 2015;43(5):1011-1021.

36. Chu DM, Ma J, Prince AL, Antony KM, Seferovic MD, Aagaard KM. Maturation of the infant microbiome community structure and function across multiple body sites and in relation to mode of delivery. Nat Med.2017;23(3):314-326.

37. Oh J, Conlan S, Polley EC, Segre JA, Kong HH. Shifts in human skin and nares microbiota of healthy children and adults. Genome Med. 2012;4(10):77.

38. Oh J, Byrd AL, Park M, NISC Comparative Sequencing Program, Kong HH, Segre JA. Temporal stability of the human skin microbiome. Cell. 2016;165(4):854-866.

39. Palmer CN, et al. Common loss-of-function variants of the epidermal barrier protein filaggrin are a major predisposing factor for atopic dermatitis. Nat Genet. 2006;38(4):441-446.

40. Paternoster L, et al. Multi-ancestry genome-wide association study of 21,000 cases and 95,000 controls identifies new risk loci for atopic dermatitis. Nat Genet. 2015;47(12):1449-1456.

41. Saunders SP, et al. Spontaneous atopic dermatitis is mediated by innate immunity, with the secondary lung inflammation of the atopic march requiring adaptive immunity. J Allergy Clin Immunol. 2016;137(2):482-491.

42. Myles IA, et al. Transplantation of human skin microbiota in models of atopic dermatitis. JCI Insight. 2016;1(10):e86955.
43. Kennedy EA, et al. Skin microbiome before development of atopic dermatitis: early colonization with commensal staphylococci at 2 months is associated with a lower risk of atopic dermatitis at 1 year. J Allergy Clin Immunol. 2017;139(1):166-172.

44. Kong HH, et al. Temporal shifts in the skin microbiome associated with disease flares and treatment in children with atopic dermatitis. Genome Res. 2012;22(5):850-859.

45. Kobayashi T, et al. Dysbiosis and Staphylococcus aureus colonization drives inflammation in atopic dermatitis. Immunity. 2015;42(4):756-766.

46. Chng KR, et al. Whole metagenome profiling reveals skin microbiome-dependent susceptibility to atopic dermatitis flare. Nat Microbiol. 2016;1(9):16106.

47. Meylan P, et al. Skin colonization by Staphylococcus aureus precedes the clinical diagnosis of atopic dermatitis in infancy. J Invest Dermatol. 2017;137(12):2497-2504.

48. Nakamura Y, et al. Staphylococcus $\delta$-toxin induces allergic skin disease by activating mast cells. Nature. 2013;503(7476):397-401.

49. Tauber M, et al. Staphylococcus aureus density on lesional and nonlesional skin is strongly associated with disease severity in atopic dermatitis. JAllergy Clin Immunol. 2016;137(4):1272-1274.e3.

50. Cogen AL, et al. Staphylococcus epidermidis antimicrobial $\delta$-toxin (phenol-soluble modulin- $\gamma$ ) cooperates with host antimicrobial peptides to kill group A Streptococcus. PLoS One. 2010;5(1):e8557.

51. Lai Y, et al. Activation of TLR2 by a small molecule produced by Staphylococcus epidermidis increases antimicrobial defense against bacterial skin infections. J Invest Dermatol. 2010;130(9):2211-2221.

52. Li D, Lei H, Li Z, Li H, Wang Y, Lai Y. A novel lipopeptide from skin commensal activates TLR2/ CD36-p38 MAPK signaling to increase antibacterial defense against bacterial infection. PLoS One. 2013;8(3):e58288.

53. Nakatsuji T, et al. Staphylococcus aureus exploits epidermal barrier defects in atopic dermatitis to trigger cytokine expression. J Invest Dermatol. 2016;136(11):2192-2200.

54. Hirasawa Y, et al. Staphylococcus aureus extracellular protease causes epidermal barrier dysfunction. J Invest Dermatol. 2010;130(2):614-617.

55. Brauweiler AM, Goleva E, Leung DYM. Th2 cytokines increase Staphylococcus aureus $\alpha$ toxininduced keratinocyte death through the signal transducer and activator of transcription 6 (STAT6). JInvest Dermatol. 2014;134(8):2114-2121.

56. Walev I, et al. Staphylococcal alpha-toxin kills human keratinocytes by permeabilizing the plasma membrane for monovalent ions. Infect Immun. 1993;61(12):4972-4979.

57. Vu AT, et al. Staphylococcus aureus membrane and diacylated lipopeptide induce thymic stromal lymphopoietin in keratinocytes through the Toll-like receptor 2-Toll-like receptor 6 pathway. J Allergy Clin Immunol. 2010;126(5):985-993.e1.

58. Savinko T, et al. Topical superantigen exposure induces epidermal accumulation of $\mathrm{CD}^{+} \mathrm{T}$ cells, a mixed Th1/Th2-type dermatitis and vigorous production of $\operatorname{IgE}$ antibodies in the murine model of atopic dermatitis. J Immunol. 2005;175(12):8320-8326. 
59. Strange P, Skov L, Lisby S, Nielsen PL, Baadsgaard O. Staphylococcal enterotoxin B applied on intact normal and intact atopic skin induces dermatitis. Arch Dermatol. 1996;132(1):27-33.

60. Venkataraman D, et al. Filaggrin loss-of-function mutations are associated with food allergy in childhood and adolescence. J Allergy Clin Immunol. 2014;134(4):876-882.e4.

61. Brough HA, et al. Peanut allergy: effect of environmental peanut exposure in children with filaggrin loss-of-function mutations. J Allergy Clin Immunol. 2014;134(4):867-875.e1.

62. Mondoulet L, et al. Intact skin and not stripped skin is crucial for the safety and efficacy of peanut epicutaneous immunotherapy (EPIT) in mice. Clin Transl Allergy. 2012;2(1):22.

63. Lack G, Fox D, Northstone K, Golding J, Avon Longitudinal Study of Parents Children Study Team. Factors associated with the development of peanut allergy in childhood. $N$ Engl J Med. 2003;348(11):977-985.

64. Noti M, et al. Exposure to food allergens through inflamed skin promotes intestinal food allergy through the thymic stromal lymphopoietin-basophil axis. J Allergy Clin Immunol. 2014;133(5):1390-1399.e1.

65. Leyva-Castillo JM, Hener P, Jiang H, Li M. TSLP produced by keratinocytes promotes allergen sensitization through skin and thereby triggers atopic march in mice. J Invest Dermatol. 2013;133(1):154-163.

66. Leyva-Castillo JM, et al. Skin thymic stromal lymphopoietin initiates Th2 responses through an orchestrated immune cascade. Nat Commun. 2013;4:2847.

67. Han H, et al. Thymic stromal lymphopoietin (TSLP)-mediated dermal inflammation aggravates experimental asthma. Mucosal Immunol. 2012;5(3):342-351.

68. Agace WW, McCoy KD. Regionalized development and maintenance of the intestinal adaptive immune landscape. Immunity. 2017;46(4):532-548.

69. Donaldson GP, Lee SM, Mazmanian SK. Gut biogeography of the bacterial microbiota. Nat Rev Microbiol. 2016;14(1):20-32.

70. Johansson ME, Phillipson M, Petersson J, Velcich A, Holm L, Hansson GC. The inner of the two Muc2 mucin-dependent mucus layers in colon is devoid of bacteria. Proc Natl Acad Sci U S A. 2008;105(39):15064-15069.

71. Gu S, et al. Bacterial community mapping of the mouse gastrointestinal tract. PLoS One. 2013;8(10):e74957.

72. Cullen TW, et al. Gut microbiota. Antimicrobial peptide resistance mediates resilience of prominent gut commensals during inflammation. Science. 2015;347(6218):170-175.

73. Zoetendal EG, et al. The human small intestinal microbiota is driven by rapid uptake and conversion of simple carbohydrates. ISME J. 2012;6(7):1415-1426.

74. Nava GM, Friedrichsen HJ, Stappenbeck TS. Spatial organization of intestinal microbiota in the mouse ascending colon. ISME J. 2011;5(4):627-638.

75. Human Microbiome Project Consortium. Structure, function and diversity of the healthy human microbiome. Nature. 2012;486(7402):207-214.

76. Qin J, et al. A human gut microbial gene cata- logue established by metagenomic sequencing. Nature. 2010;464(7285):59-65.

77. Hong PY, Croix JA, Greenberg E, Gaskins HR, Mackie RI. Pyrosequencing-based analysis of the mucosal microbiota in healthy individuals reveals ubiquitous bacterial groups and micro-heterogeneity. PLoS One. 2011;6(9):e25042.

78. Yassour M, et al. Natural history of the infant gut microbiome and impact of antibiotic treatment on bacterial strain diversity and stability. Sci Transl Med. 2016;8(343):343ra81.

79. Bäckhed F, et al. Dynamics and stabilization of the human gut microbiome during the first year of life. Cell Host Microbe. 2015;17(5):690-703.

80. Rogier EW, et al. Secretory antibodies in breast milk promote long-term intestinal homeostasis by regulating the gut microbiota and host gene expression. Proc Natl Acad Sci U S A. 2014;111(8):3074-3079.

81. Yu ZT, Chen C, Newburg DS. Utilization of major fucosylated and sialylated human milk oligosaccharides by isolated human gut microbes. Glycobiology. 2013;23(11):1281-1292.

82. Yatsunenko T, et al. Human gut microbiome viewed across age and geography. Nature. 2012;486(7402):222-227.

83. David LA, et al. Diet rapidly and reproducibly alters the human gut microbiome. Nature. 2014;505(7484):559-563.

84. Martínez I, Muller CE, Walter J. Long-term temporal analysis of the human fecal microbiota revealed a stable core of dominant bacterial species. PLoS One. 2013;8(7):e69621.

85. Faith JJ, et al. The long-term stability of the human gut microbiota. Science. 2013;341(6141):1237439.

86. Mullineaux-Sanders C, Suez J, Elinav E, Frankel G. Sieving through gut models of colonization resistance. Nat Microbiol. 2018;3(2):132-140.

87. Lathrop SK, et al. Peripheral education of the immune system by colonic commensal microbiota. Nature. 2011;478(7368):250-254.

88. Geuking MB, et al. Intestinal bacterial colonization induces mutualistic regulatory $\mathrm{T}$ cell responses. Immunity. 2011;34(5):794-806

89. Round JL, Mazmanian SK. Inducible Foxp $3^{+}$ regulatory T-cell development by a commensal bacterium of the intestinal microbiota. Proc Natl Acad Sci U S A. 2010;107(27):12204-12209.

90. Dasgupta S, Erturk-Hasdemir D, Ochoa-Reparaz J, Reinecker HC, Kasper DL. Plasmacytoid dendritic cells mediate anti-inflammatory responses to a gut commensal molecule via both innate and adaptive mechanisms. Cell Host Microbe. 2014;15(4):413-423.

91. Atarashi K, et al. Treg induction by a rationally selected mixture of Clostridia strains from the human microbiota. Nature. 2013;500(7461):232-236.

92. Atarashi $\mathrm{K}$, et al. Induction of colonic regulatory $\mathrm{T}$ cells by indigenous Clostridium species. Science. 2011;331(6015):337-341.

93. Sefik E, et al. Mucosal immunology. Individual intestinal symbionts induce a distinct population of $\mathrm{ROR} \gamma^{+}$regulatory T cells. Science. 2015;349(6251):993-997.

94. Ohnmacht C, et al. Mucosal immunology. The microbiota regulates type 2 immunity through ROR $\gamma \mathrm{t}^{+}$T cells. Science. 2015;349(6251):989-993.

95. Smith PM, et al. The microbial metabolites, short-chain fatty acids, regulate colonic Treg cell homeostasis. Science. 2013;341(6145):569-573.

96. Trompette A, et al. Gut microbiota metabolism of dietary fiber influences allergic airway disease and hematopoiesis. Nat Med. 2014;20(2):159-166.

97. Maslowski KM, et al. Regulation of inflammatory responses by gut microbiota and chemoattractant receptor GPR43. Nature. 2009;461(7268):1282-1286.

98. Singh N, et al. Activation of Gpr109a, receptor for niacin and the commensal metabolite butyrate, suppresses colonic inflammation and carcinogenesis. Immunity. 2014;40(1):128-139.

99. Arpaia N, et al. Metabolites produced by commensal bacteria promote peripheral regulatory T-cell generation. Nature. 2013;504(7480):451-455.

100. Furusawa Y, et al. Commensal microbe-derived butyrate induces the differentiation of colonic regulatory T cells. Nature. 2013;504(7480):446-450.

101. Roediger WE. Role of anaerobic bacteria in the metabolic welfare of the colonic mucosa in man. Gut.1980;21(9):793-798.

102. Byndloss MX, et al. Microbiota-activated PPAR- $\gamma$ signaling inhibits dysbiotic Enterobacteriaceae expansion. Science. 2017;357(6351):570-575.

103. Miyamoto J, et al. A gut microbial metabolite of linoleic acid, 10-hydroxy-cis-12-octadecenoic acid, ameliorates intestinal epithelial barrier impairment partially via GPR40-MEK-ERK pathway. J Biol Chem. 2015;290(5):2902-2918.

104.Zelante T, et al. Tryptophan catabolites from microbiota engage aryl hydrocarbon receptor and balance mucosal reactivity via interleukin-22. Immunity. 2013;39(2):372-385.

105. Brestoff JR, Artis D. Commensal bacteria at the interface of host metabolism and the immune system. Nat Immunol. 2013;14(7):676-684.

106. Kinoshita M, et al. Dietary folic acid promotes survival of Foxp3+ regulatory T cells in the colon. Jimmunol. 2012;189(6):2869-2878.

107. Kunisawa J, Hashimoto E, Ishikawa I, Kiyono H. A pivotal role of vitamin $\mathrm{B} 9$ in the maintenance of regulatory $\mathrm{T}$ cells in vitro and in vivo. PLoS One. 2012;7(2):e32094.

108. Magnúsdóttir S, Ravcheev D, de Crécy-Lagard V, Thiele I. Systematic genome assessment of B-vitamin biosynthesis suggests co-operation among gut microbes. Front Genet. 2015;6:148.

109. Sudo N, Sawamura S, Tanaka K, Aiba Y, Kubo C, Koga Y. The requirement of intestinal bacterial flora for the development of an IgE production system fully susceptible to oral tolerance induction. JImmunol. 1997;159(4):1739-1745.

110. Ishikawa $\mathrm{H}$, et al. Effect of intestinal microbiota on the induction of regulatory $\mathrm{CD} 25^{+} \mathrm{CD} 4^{+} \mathrm{T}$ cells. Clin Exp Immunol. 2008;153(1):127-135.

111. Noval Rivas M, et al. A microbiota signature associated with experimental food allergy promotes allergic sensitization and anaphylaxis. J Allergy Clin Immunol. 2013;131(1):201-212.

112. Azad MB, et al. Infant gut microbiota and food sensitization: associations in the first year of life. Clin Exp Allergy. 2015;45(3):632-643.

113. Berni Canani R, et al. Lactobacillus rhamnosus GG-supplemented formula expands butyrateproducing bacterial strains in food allergic infants. ISME J. 2016;10(3):742-750.

114. Björkstén B, Naaber P, Sepp E, Mikelsaar M. The 
intestinal microflora in allergic Estonian and Swedish 2-year-old children. Clin Exp Allergy. 1999;29(3):342-346.

115. Ling Z, et al. Altered fecal microbiota composition associated with food allergy in infants. Appl Environ Microbiol. 2014;80(8):2546-2554.

116. Feehley T, et al. Healthy infants harbor intestinal bacteria that protect against food allergy [published online ahead of print January 14, 2019]. Nat Med.https://doi.org/10.1038/s41591-018-0324-z.

117. Stefka AT, et al. Commensal bacteria protect against food allergen sensitization. Proc Natl Acad Sci U S A. 2014;111(36):13145-13150.

118. Tan J, et al. Dietary fiber and bacterial SCFA enhance oral tolerance and protect against food allergy through diverse cellular pathways. Cell Rep. 2016;15(12):2809-2824.

119. Ventura MT, et al. Intestinal permeability in patients with adverse reactions to food. Dig Liver Dis. 2006;38(10):732-736.

120. Bashir ME, Louie S, Shi HN, Nagler-Anderson C. Toll-like receptor 4 signaling by intestinal microbes influences susceptibility to food allergy. J Immunol. 2004;172(11):6978-6987.

121. Vatanen $\mathrm{T}$, et al. Variation in microbiome LPS immunogenicity contributes to autoimmunity in humans. Cell. 2016;165(4):842-853.

122.Fujimura KE, et al. Neonatal gut microbiota associates with childhood multisensitized atopy and $\mathrm{T}$ cell differentiation. Nat Med. 2016;22(10):1187-1191.

123. Arrieta MC, et al. Early infancy microbial and metabolic alterations affect risk of childhood asthma. Sci Transl Med.2015;7(307):307ra152.

124. Thorburn AN, et al. Evidence that asthma is a developmental origin disease influenced by maternal diet and bacterial metabolites. Nat Commun. 2015;6:7320.

125. Thio CL, Chi PY, Lai AC, Chang YJ. Regulation of type 2 innate lymphoid cell-dependent airway hyperreactivity by butyrate. JAllergy Clin Immunol. 2018;142(6):1867-1883.e12.

126. Marsland BJ. Influences of the microbiome on the early origins of allergic asthma. Ann Am Thorac Soc. 2013;10(suppl):S165-S169.

127. Hilty M, et al. Disordered microbial communities in asthmatic airways. PLoS One. 2010;5(1):e8578.

128. Arrieta MC, Finlay B. The intestinal microbiota and allergic asthma. J Infect. 2014;69(suppl 1):S53-S55.

129. Noval Rivas M, Crother TR, Arditi M. The microbiome in asthma. Curr Opin Pediatr. 2016;28(6):764-771.

130. Dickson RP, et al. Spatial variation in the healthy human lung microbiome and the adapted island model of lung biogeography. Ann Am Thorac Soc. 2015;12(6):821-830.

131. Charlson ES, et al. Topographical continuity of bacterial populations in the healthy human respiratory tract. Am J Respir Crit Care Med. 2011;184(8):957-963.

132. Teo SM, et al. Airway microbiota dynamics uncover a critical window for interplay of pathogenic bacteria and allergy in childhood respiratory disease. Cell Host Microbe. 2018;24(3):341-352.e5.

133. Biesbroek $\mathrm{G}$, et al. Early respiratory microbiota composition determines bacterial succession patterns and respiratory health in children. Am J Respir Crit Care Med. 2014;190(11):1283-1292.
134. Huang YJ, et al. The airway microbiome in patients with severe asthma: associations with disease features and severity. J Allergy Clin Immunol. 2015;136(4):874-884.

135. Huang YJ, et al. Airway microbiota and bronchial hyperresponsiveness in patients with suboptimally controlled asthma. JAllergy Clin Immunol. 2011;127(2):372-381.e1.

136. Bisgaard $\mathrm{H}$, et al. Childhood asthma after bacterial colonization of the airway in neonates. $N$ Engl J Med. 2007;357(15):1487-1495.

137. Marri PR, Stern DA, Wright AL, Billheimer D, Martinez FD. Asthma-associated differences in microbial composition of induced sputum. J Allergy Clin Immunol. 2013;131(2):346-352.e1.

138. Teo SM, et al. The infant nasopharyngeal microbiome impacts severity of lower respiratory infection and risk of asthma development. Cell Host Microbe. 2015;17(5):704-715.

139. Kloepfer KM, et al. Detection of pathogenic bacteria during rhinovirus infection is associated with increased respiratory symptoms and asthma exacerbations. J Allergy Clin Immunol. 2014;133(5):1301-1307.e1.

140. Green BJ, et al. Potentially pathogenic airway bacteria and neutrophilic inflammation in treatment resistant severe asthma. PLoS One. 2014;9(6):e100645.

141. Lambrecht BN, Hammad H. The immunology of asthma. Nat Immunol. 2015;16(1):45-56.

142.Jackson DJ, et al. Wheezing rhinovirus illnesses in early life predict asthma development in high-risk children. Am J Respir Crit Care Med. 2008;178(7):667-672.

143. Rubner FJ, et al. Early life rhinovirus wheezing, allergic sensitization, and asthma risk at adolescence. J Allergy Clin Immunol. 2017;139 (2):501-507.

144. Beale J, et al. Rhinovirus-induced IL-25 in asthma exacerbation drives type 2 immunity and allergic pulmonary inflammation. Sci Transl Med. 2014;6(256):256ra134.

145. Jackson DJ, et al. IL-33-dependent type 2 inflammation during rhinovirus-induced asthma exacerbations in vivo. Am J Respir Crit Care Med. 2014;190(12):1373-1382.

146. Hong JY, et al. Neonatal rhinovirus induces mucous metaplasia and airways hyperresponsiveness through IL-25 and type 2 innate lymphoid cells. J Allergy Clin Immunol. 2014;134(2):429-439.

147. Han M, et al. The innate cytokines IL-25, IL-33, and TSLP cooperate in the induction of type 2 innate lymphoid cell expansion and mucous metaplasia in rhinovirus-infected immature mice. J Immunol. 2017;199(4):1308-1318.

148.Wesemann DR, Nagler CR. The microbiome, timing, and barrier function in the context of allergic disease. Immunity. 2016;44(4):728-738.

149. Hirsch AG, et al. Early-life antibiotic use and subsequent diagnosis of food allergy and allergic diseases. Clin Exp Allergy. 2017;47(2):236-244.

150. Herbst T, et al. Dysregulation of allergic airway inflammation in the absence of microbial colonization. Am J Respir Crit Care Med. 2011;184(2):198-205.

151. Cahenzli J, Köller Y, Wyss M, Geuking MB, McCoy KD. Intestinal microbial diversity during early-life colonization shapes long-term IgE levels. Cell Host Microbe. 2013;14(5):559-570.
152. Olszak T, et al. Microbial exposure during early life has persistent effects on natural killer T cell function. Science. 2012;336(6080):489-493.

153. Perkin MR, et al. Randomized trial of introduction of allergenic foods in breast-fed infants. N Engl J Med. 2016;374(18):1733-1743.

154. Du Toit G, et al. Randomized trial of peanut consumption in infants at risk for peanut allergy. N Engl J Med. 2015;372(9):803-813.

155. Du Toit G, et al. Effect of avoidance on peanut allergy after early peanut consumption. $N$ EnglJ Med. 2016;374(15):1435-1443.

156.West CE, Dzidic M, Prescott SL, Jenmalm MC. Bugging allergy; role of pre-, pro- and synbiotics in allergy prevention. Allergol Int. 2017;66(4):529-538.

157. Gomez de Agüero M, et al. The maternal microbiota drives early postnatal innate immune development. Science. 2016;351(6279):1296-1302.

158. Thome JJ, et al. Early-life compartmentalization of human $\mathrm{T}$ cell differentiation and regulatory function in mucosal and lymphoid tissues. Nat Med. 2016;22(1):72-77.

159. Hebel $\mathrm{K}$, et al. $\mathrm{CD} 4^{+} \mathrm{T}$ cells from human neonates and infants are poised spontaneously to run a nonclassical IL-4 program. J Immunol. 2014;192(11):5160-5170.

160. Webster RB, Rodriguez Y, Klimecki WT, Vercelli D. The human IL-13 locus in neonatal $\mathrm{CD}^{+} \mathrm{T}$ cells is refractory to the acquisition of a repressive chromatin architecture. J Biol Chem. 2007;282(1):700-709.

161. Pannaraj PS, et al. Association between breast milk bacterial communities and establishment and development of the infant gut microbiome. JAMA Pediatr. 2017;171(7):647-654.

162.Rajani PS, Seppo AE, Järvinen KM. Immunologically active components in human milk and development of atopic disease, with emphasis on food allergy, in the pediatric population. Front Pediatr. 2018;6:218.

163. Oddy WH, Rosales F. A systematic review of the importance of milk TGF- $\beta$ on immunological outcomes in the infant and young child. Pediatr Allergy Immunol. 2010;21(1 pt 1):47-59.

164. Tulic MK, et al. Differences in innate immune function between allergic and nonallergic children: new insights into immune ontogeny. J Allergy Clin Immunol.2011;127(2):470-478.e1.

165.Zhang Y, et al. Cord blood monocyte-derived inflammatory cytokines suppress IL-2 and induce nonclassic " $\mathrm{T}(\mathrm{H}) 2$-type" immunity associated with development of food allergy. Sci Transl Med. 2016;8(321):321ra8.

166. Dominguez-Bello MG, et al. Partial restoration of the microbiota of cesarean-born infants via vaginal microbial transfer. Nat Med. 2016;22(3):250-253.

167. Forsberg A, West CE, Prescott SL, Jenmalm MC. Pre- and probiotics for allergy prevention: time to revisit recommendations? Clin Exp Allergy. 2016;46(12):1506-1521.

168. Fiocchi A, et al. World Allergy OrganizationMcMaster University Guidelines for Allergic Disease Prevention (GLAD-P): Probiotics. World Allergy Organ J. 2015;8(1):4.

169.O'Toole PW, Marchesi JR, Hill C. Next-generation probiotics: the spectrum from probiotics to live biotherapeutics. Nat Microbiol. 2017;2:17057. 